\title{
Magnetic field dissipation in neutron star crusts: from magnetars to isolated neutron stars
}

\author{
J. A. Pons and U. Geppert \\ Departament de Física Aplicada, Universitat d'Alacant, Ap. Correus 99, 03080 Alacant, Spain \\ e-mail: Jose.A.Pons@ua.es
}

Received 12 March 2007 / Accepted 8 April 2007

\begin{abstract}
Context. We study the non-linear evolution of magnetic fields in neutron star crusts with special attention to the influence of the Hall drift.

Aims. Our goal is to understand the conditions for fast dissipation due to the Hall term in the induction equation. We study the interplay of Ohmic dissipation and Hall drift in order to find a timescale for the overall crustal field decay.

Methods. We solve the Hall induction equation numerically by means of a hybrid method (spectral in angles but finite differences in the radial coordinate). The microphysical input consists of the most modern available crustal equation of state, composition, and electrical conductivities.

Results. We present the first long-term simulations of the non-linear magnetic field evolution in realistic neutron star crusts with a stratified electron number density and temperature dependent conductivity. We show that Ohmic dissipation influenced by Hall drift takes place in neutron star crusts on a timescale of $10^{6}$ years. When the initial magnetic field has magnetar strength, the fast Hall drift results in an initial rapid dissipation stage that lasts $\sim 10^{4}$ years. The interplay of the Hall drift with the temporal variation and spatial gradient of conductivity tends to favor the displacement of toroidal fields toward the inner crust, where stable configurations can last for $\sim 10^{6}$ years. We show that the thermally emitting, isolated neutron stars, such as the Magnificent Seven, are very likely descendants of neutron stars born as magnetars.
\end{abstract}

Key words. stars: neutron - stars: magnetic fields - stars: evolution

\section{Introduction}

Since the early days of neutron star (NS) research, when a first estimate of the characteristic decay time of its magnetic field was performed (Baym et al. 1969), our models of magnetic field evolution in isolated NSs has become more and more complex. However, a complete theoretical model that explains all observational facts satisfactorily does not exist yet. There is very general agreement that the magnetic field in ultra-magnetized NSs (magnetars) decays on timescales of $10^{3}-10^{5}$ years (Woods \& Thompson 2006; Harding \& Lai 2006). Very recently, it has been argued (Pons et al. 2007) that field decay may also be an effective heating mechanism in isolated NSs, which are somewhat less magnetized than standard magnetars (AXPs and SGRs). The observed correlation between surface temperatures and magnetic field strengths is evidence of efficient dissipation, hence Joule heating, near the surface layers of NSs. In addition, evidence of braking indexes significantly larger than 3 , which are inferred for an ensemble of a dozen of pulsars in an active age of a few $10^{5}$ years, indicate that epochs of rapid decay in relatively young NSs occur (Johnston \& Galloway 1999; Geppert \& Rheinhardt 2002).

Another proof of rapid magnetic field evolution in the subsurface crustal layers would be the observation of small structures (significantly smaller than the dipolar mode) close to the surface. These small-scale structures could not be survivors from the magnetic field structure acquired by the NS at birth; their small scales, in combination with the low electric conductivity in the outer crust, would have erased such field structures on timescales shorter than 1 Myr. In addition, the growing understanding of the physics of the drifting sub-pulses supports the idea that small-scale crustal field structures must be present (Gil et al. 2003, 2006). The existence of strong crustal fields in NSs as old as about $1 \mathrm{Myr}$ is also necessary to create the nonuniform surface temperature distribution observed e.g. in the "Magnificent Seven" (see Pons et al. 2002; Schwope et al. 2005; Haberl et al. 2006 for the observational evidence and Geppert et al. 2004, 2006; Pérez-Azorín et al. 2006a,b for the theoretical interpretation). All this phenomena can only be understood if there is a crustal magnetic field with a strong toroidal component and with more structure than the simple dipolar model.

On the other hand, population synthesis studies suggest that old pulsars show no significant magnetic field decay over their life time, i.e. the decay time must be longer than $10 \mathrm{Myr}$ (Hartman et al. 1997; Regimbau \& de Freitas Pacheco 2001), although the opposite conclusion has also been claimed (Gonthier et al. 2004). These, at first glance, contradictory facts can be satisfactorily resolved by the (quite natural) assumption that the NS magnetic field is maintained by two current systems. Long living currents in the superconducting core support the largescale dipolar field and are responsible for the spin down of old pulsars. Currents in the crust support the short-lived part of the field. It decays on a timescale of $10^{5}-10^{7}$ years, depending on the conductivity, thickness of the crust, and the strength and structure of the initial field. Estimates of how fast a core-anchored field could be expelled and subsequently dissipated in the crust result in characteristic timescales exceeding $100 \mathrm{Myr}$ (Konenkov $\&$ Geppert 2001). The dipolar component of the crust is superimposed, outside the NS, to the core component. Depending on 
their relative strengths, a rapid decay of the crustal dipolar field may or may not have observable influence on the pulsar spin down behavior.

Besides the Ohmic diffusion, which will proceed fast in the outermost low-density crustal regions and during the early hot phase of a NS's life, another process that can change the crustal field structure, both quantitatively and qualitatively, is the Hall drift. During the last two decades, many authors have studied the effects of Hall drift onto the evolution of magnetic fields in isolated NSs (Haensel et al. 1990; Goldreich \& Reisenegger 1992; Muslimov 1994; Naito \& Kojima 1994; Urpin \& Shalybkov 1995; Shalybkov \& Urpin 1997; Vainshtein et al. 2000; Rheinhardt \& Geppert 2002; Geppert \& Rheinhardt 2002; Hollerbach \& Ruediger 2002; Geppert et al. 2003; Rheinhardt et al. 2004; Cumming et al. 2004). By use of the analogy of the Hall induction equation with the vorticity equation of an incompressible liquid, Goldreich \& Reisenegger (1992) developed the idea of the Hall cascade. It transfers magnetic energy from the largest to smaller scales until a critical scalelength is reached below which Ohmic decay becomes dominant. Vainshtein et al. (2000) considered the Hall drift for the first time in a stratified NS crust. In the presence of a density profile, Hall currents are able to create current sheets, which are places where very efficient dissipation occurs. This effect is dramatic if current sheets are located just below the surface, where the conductivity is lower, but even if the drift is directed towards the highly conductive inner crust, the small scale of the locally intense field causes a significantly faster dissipation of magnetic energy than the purely Ohmic diffusion estimate. In summary, this MHD-like process (considering the crust as a one-component plasma) is by itself energy conserving. However, it affects the magnetic field by two inherent tendencies: the creation of small-scale field structures by transferring magnetic energy from the initial largescale field, and the drift of growing structures towards a region where current sheets are created.

Another interesting effect of the Hall drift has been proposed by Rheinhardt \& Geppert (2002). They show, by means of a stability analysis of the linearized Hall induction equation, that the transfer of energy from the large-scale (background) field to the small-scale modes may proceed in a non-local way in the momentum space, resulting in a Hall instability. Cumming et al. (2004) show that the growth rate of this instability depends on the shear in the velocity of the electrons whose current supports the background field. As they point out, it is unclear whether the Hall instability is relevant for the field evolution in the crust, since the Hall cascade may proceed sufficiently fast to fill in the intermediate scales between the large-scale initial field and the unstable growing small-scale ones.

In the present study we intend to consider the field evolution as realistic as possible. Thus, we will solve the non-linear Hall induction equation numerically in the crustal region, using a crustal density and conductivity profile according to the stateof-the-art microphysical input. We start with initial field configurations, which consist both of poloidal and toroidal field components that fulfill boundary conditions at the superconducting core and at the surface. We follow the cooling history of a NS, starting with an initially hot NS where the magnetization parameter is relatively small and the field evolution in the crust is almost completely determined by Ohmic diffusion. As the NS cools down, the magnetization parameter increases, thereby gradually enhancing the relative importance of the Hall drift. Our aim is to reach a better understanding of the effects that Hall drift may have on the crustal field evolution and its consequences for the rotational and thermal evolution of isolated NSs.
The paper is organized as follows. In Sect. 2 we present the Hall induction equation, where the magnetic field is represented in terms of its poloidal and toroidal components that are expanded in a series of spherical harmonics. Section 3 is devoted to purely toroidal fields. For this case we show that the induction equation can be written in a form similar to the Burgers equation. This allows us to discuss the importance of the field-dependent drift in a clear way. In Sect. 4 we explain the input microphysics, the NS model, and the initial conditions. In Sect. 5 we present the results for different initial field configurations. Finally, in Sect. 6, we discuss our main conclusions and give an outlook for future improvements.

\section{Basic equations and formalism}

In the crystallized crust of NSs, where convective motions of the conductive material play no role, the evolution of the magnetic field is governed by the Hall-induction equation

$\frac{\partial \boldsymbol{B}}{\partial t}=-\frac{c^{2}}{4 \pi} \nabla \times\left(\frac{1}{\sigma}\left\{\nabla \times \boldsymbol{B}+\omega_{B} \tau[(\nabla \times \boldsymbol{B}) \times \boldsymbol{b}]\right\}\right)$,

where $\boldsymbol{b}$ is the unit vector in the direction of the magnetic field $\boldsymbol{b}=\boldsymbol{B} / B, B$ the magnetic field strength, $\tau$ the relaxation time of the electrons, and $\omega_{B}=e B / m_{\mathrm{e}}^{*} c$ the electron cyclotron frequency. Here, $\sigma=e^{2} n_{\mathrm{e}} \tau / m_{\mathrm{e}}^{*}$ is the electrical conductivity parallel to the magnetic field, and $n_{\mathrm{e}}$ the electron number density. The Hall drift term (the term proportional to $\omega_{B} \tau$ ) at the r.h.s. of Eq. (1) is a consequence of the Lorentz force acting on the electrons. The tensor components of the electric conductivity are derived in the relaxation time approximation (Yakovlev \& Shalybkov 1991). If the magnetization parameter $\omega_{B} \tau$ significantly exceeds unity, the Hall drift dominates, which results in a very different field evolution from the purely Ohmic case. A large magnetization parameter, typically $\approx 1000$ during some stages (Geppert \& Rheinhardt 2002; Pérez-Azorín et al. 2006), strongly suppresses the electric conductivity perpendicular to the magnetic field.

The limited success solving Eq. (1) up to now has been restricted to a magnetization parameter not exceeding 200 (Hollerbach \& Rüdiger 2002) ${ }^{1}$. These numerical limitations led us to use an alternative approach, since fully spectral codes systematically have unsurmountable problems dealing with structures where discontinuities or very large gradients of the variables appear. This will happen, for example, if current sheets develop as a consequence of the Hall drift. The next section will describe in detail why spectral methods may not be well-suited to dealing with this problem. We have decided to employ a semispectral method that describes the angular part of the field by spherical harmonics but uses a spatial difference scheme in radial direction.

The formalism that uses the representation of fields by their poloidal and toroidal parts and their expansion in a series of spherical harmonics was developed by Rädler (1973). This formalism allows transformation of the (vector) induction equation into two scalar equations. The magnetic field is decomposed into poloidal and toroidal components:

$\boldsymbol{B}=\boldsymbol{B}_{\text {pol }}+\boldsymbol{B}_{\text {tor }}$

Hereafter, we follow the notation of Geppert \& Wiebicke (1991). The two components are described by two functions, $\Phi(r, \theta, \varphi, t)$

1 Their parameter $R_{B}$ is equivalent to our $\omega_{B} \tau$ calculated for a fixed, initial magnetic field strength $B_{0}$. 
and $\Psi(r, \theta, \varphi, t)$, where $r, \theta$, and $\varphi$ are the usual spherical coordinates. Explicitly, the components of the field are given by

$$
\begin{aligned}
& \boldsymbol{B}_{\mathrm{pol}}=\nabla \times \boldsymbol{A}, \quad \boldsymbol{A}=-\boldsymbol{r} \times \nabla \Phi, \\
& \boldsymbol{B}_{\mathrm{pol}}=-\boldsymbol{r} \nabla^{2} \Phi+\nabla\left(\frac{\partial(r \Phi)}{\partial r}\right), \\
& \boldsymbol{B}_{\text {tor }}=-\boldsymbol{r} \times \nabla \Psi .
\end{aligned}
$$

This formulation has the advantage of automatically fulfilling the divergence condition $(\nabla \cdot \boldsymbol{B}=0)$. Inserting the expressions of Eq. (3) into Eq. (1), we arrive at two partial differential equations describing the evolution of the poloidal and of the toroidal part of the magnetic field:

$$
\begin{aligned}
& \frac{\partial \Phi}{\partial t}=\frac{1}{\hat{\sigma}} \nabla^{2} \Phi+D, \\
& \frac{\partial \Psi}{\partial t}=\frac{1}{\hat{\sigma}}\left(\nabla^{2} \Psi-\frac{1}{r} \frac{\partial \log \hat{\sigma}}{\partial r} \frac{\partial(r \Psi)}{\partial r}\right)+C .
\end{aligned}
$$

Here, $\hat{\sigma}=4 \pi \sigma / c^{2}$ and $D$ and $C$ stand for the nonlinear terms describing the Hall drift and coupling both components (poloidal and toroidal) of the magnetic field. We have assumed that the conductivity only depends on the radial coordinate and is independent of the magnetic field strength.

After expanding the functions $\Phi$ and $\Psi$ in a series of spherical harmonics

$\Phi=\frac{1}{r} \sum_{n, m} \Phi_{n m}(r, t) Y_{n m}(\theta, \phi)$,

$\Psi=\frac{1}{r} \sum_{n, m} \Psi_{n m}(r, t) Y_{n m}(\theta, \phi)$,

where $n=1, \ldots, n_{\max }$ and $m=-n, \ldots,+n$. The vector potential, as well as the poloidal and toroidal parts of the magnetic field, can be written as

$$
\begin{aligned}
& \boldsymbol{A}=-\left(\frac{1}{r} \sum_{n, m} \Phi_{n m} \frac{\mathrm{d} Y_{n m}}{\mathrm{~d} \theta}\right) \boldsymbol{e}_{\phi}, \\
& \boldsymbol{B}_{\mathrm{pol}}=\frac{1}{r^{2}} \sum_{n, m} n(n+1) \Phi_{n m} Y_{n m} \boldsymbol{e}_{r}+\frac{1}{r} \sum_{n, m} \frac{\partial \Phi_{n m}}{\partial r} \frac{\mathrm{d} Y_{n m}}{\mathrm{~d} \theta} \boldsymbol{e}_{\theta}, \\
& \boldsymbol{B}_{\text {tor }}=-\left(\frac{1}{r} \sum_{n, m} \Psi_{n m} \frac{\mathrm{d} Y_{n m}}{\mathrm{~d} \theta}\right) \boldsymbol{e}_{\phi},
\end{aligned}
$$

while the corresponding components of the current density, given by $\boldsymbol{J}=\frac{c}{4 \pi} \nabla \times \boldsymbol{B}$, is

$$
\begin{aligned}
\frac{4 \pi}{c} \boldsymbol{J}= & \frac{1}{r^{2}} \sum_{n, m} n(n+1) \Psi_{n m} Y_{n m} \boldsymbol{e}_{r}+\frac{1}{r} \sum_{n, m} \frac{\partial \Psi_{n m}}{\partial r} \frac{\mathrm{d} Y_{n m}}{\mathrm{~d} \theta} \boldsymbol{e}_{\theta} \\
& +\frac{1}{r} \sum_{n, m}\left[\frac{\partial^{2} \Phi_{n m}}{\partial r^{2}}-\frac{n(n+1)}{r^{2}} \Phi_{n m}\right] \frac{\mathrm{d} Y_{n m}}{\mathrm{~d} \theta} \boldsymbol{e}_{\phi} .
\end{aligned}
$$

Finally, by inserting the expansions of Eq. (6) into Eq. (1), we arrive at an infinite set of partial differential equations:

$$
\begin{aligned}
& \frac{\partial \Phi_{n m}}{\partial t}=\frac{1}{\hat{\sigma}}\left(\frac{\partial^{2} \Phi_{n m}}{\partial r^{2}}-\frac{n(n+1)}{r^{2}} \Phi_{n m}\right)+D_{n m}, \\
& \frac{\partial \Psi_{n m}}{\partial t}=\frac{1}{\hat{\sigma}}\left(\frac{\partial^{2} \Psi_{n m}}{\partial r^{2}}-\frac{n(n+1)}{r^{2}} \Psi_{n m}-\frac{1}{\hat{\sigma}} \frac{\partial \hat{\sigma}}{\partial r} \frac{\partial \Psi_{n m}}{\partial r}\right)+C_{n m} .
\end{aligned}
$$

In this paper we restrict ourselves to axially symmetric field configurations, i.e., the index $m=0$, and we drop it henceforth.
Following the derivation of Geppert \& Wiebicke (1991), the nonlinear coupling terms are

$$
\begin{aligned}
D_{n}= & \frac{\hat{\tau}}{\hat{\sigma} r^{2}} \sum_{k, k^{\prime}} I^{(2)}\left(\frac{\partial}{\partial r} \Phi_{k} \Psi_{k^{\prime}}-\frac{\partial}{\partial r} \Psi_{k} \Phi_{k^{\prime}}\right), \\
C_{n}= & \sum_{k, k^{\prime}} I^{(2)} \frac{\partial}{\partial r}\left(\frac{\hat{\tau}}{\hat{\sigma} r^{2}}\left[\Psi_{k} \Psi_{k^{\prime}}+\Phi_{k}^{(1)} \Phi_{k^{\prime}}\right]\right) \\
& +\frac{\hat{\tau}}{\hat{\sigma} r^{2}} \sum_{k, k^{\prime}} I^{(3)}\left[\Psi_{k} \frac{\partial}{\partial r} \Psi_{k^{\prime}}+\frac{\partial}{\partial r} \Phi_{k} \Phi_{k^{\prime}}^{(1)}\right],
\end{aligned}
$$

where

$\Phi_{k}^{(1)}=\left(\frac{\partial^{2}}{\partial r^{2}}-\frac{k(k+1)}{r^{2}}\right) \Phi_{k}$.

Above, $\hat{\tau}=\omega_{B} \tau / B$, and $I^{(2)}, I^{(3)}$ are expressions that contain the Clebsch-Gordan coefficients that reflect the coupling properties of the field modes with different multipolarity (see Eq. (60) in Geppert \& Wiebicke 1991).

Using the orthonormality properties of the spherical harmonics, the volume-integrated magnetic energy can be calculated as

$$
\begin{aligned}
& \frac{1}{8 \pi} \int \mathrm{d} V B^{2}= \\
& \frac{1}{8 \pi} \int \mathrm{d} r \sum_{n} n(n+1)\left[n(n+1)\left(\frac{\Phi_{n}}{r}\right)^{2}+\left(\frac{\mathrm{d} \Phi_{n}}{\mathrm{~d} r}\right)^{2}+\Psi_{n}^{2}\right] .
\end{aligned}
$$

As a criterion for the magnetic energy conservation we check that the equality

$\frac{1}{8 \pi} \frac{\mathrm{d}}{\mathrm{d} t} \int B^{2} \mathrm{~d} V=-\int \frac{J^{2}}{\sigma} \mathrm{d} V$

is satisfied during the evolution within a certain tolerance (typically less than $10^{-3}$ ). The integral on the 1.h.s. is evaluated over the volume occupied by the magnetic field (including the vacuum exterior region), while the integral on the r.h.s. is calculated over the region where currents exist (the crust).

\subsection{Outer boundary conditions}

Since we consider realistic crusts with finite electric conductivity in this study, surface currents are excluded. This means that we require all components of the magnetic field to be continuous across the NS surface, i.e. that the scalar fields $\Phi_{n}$ and $\Psi_{n}$, and the derivative $\frac{\partial \Phi_{n}}{\partial r}$, are continuous through the outer boundary (see also Rädler 1973).

The external vacuum solution of a NS magnetic field is determined by $\nabla \times \boldsymbol{B}=0, \nabla \cdot \boldsymbol{B}=0$, and the boundary conditions. From Eq. (7), the vanishing curl condition leads to

$\frac{1}{r} \sum_{n}\left[\frac{\partial^{2} \Phi_{n}}{\partial r^{2}}-\frac{n(n+1)}{r^{2}} \Phi_{n}\right] \frac{\mathrm{d} Y_{n}}{\mathrm{~d} \theta} \boldsymbol{e}_{\phi}=0$ at $r \geq R$,

which can be expressed as $\Delta \Phi=0$, where $\Delta$ is the Laplacian. The index $m=0$ in $Y_{n m}$ has been omitted for simplicity. The only physical solution of this equation is $\Phi_{n}=a_{n} r^{-n}$. Therefore, the requirement of continuity across the surface results in

$\frac{\partial \Phi_{n}}{\partial r}=-\frac{n}{R} \Phi_{n}$ at $r=R$ 
Because the poloidal current must also vanish in vacuum, we can derive another general boundary condition:

$$
\sum_{n}\left[\frac{\Psi_{n}}{r \sin \theta} \frac{\partial\left(\sin \theta \frac{\partial Y_{n}}{\partial \theta}\right)}{\partial \theta} \boldsymbol{e}_{\boldsymbol{r}}+\frac{1}{r} \frac{\partial\left(r \Psi_{n} \frac{\partial Y_{n}}{\partial \theta}\right)}{\partial r} \boldsymbol{e}_{\theta}\right]=0 \text { for } r \geq R
$$

The existence of surface currents may affect the condition on the $\theta$-component of the poloidal current density, but radial currents penetrating into the vacuum cannot exist. Thus the outer boundary condition for the toroidal field is simply

$\Psi_{n}=0$ at $r=R$.

In general, the poloidal tangential $\theta$-component of the current density has to vanish at the surface only if the electric conductivity vanishes there. Then, according to Ohm's law, any finite tangential current density would cause an infinite tangential electrical field that contradicts the energy conservation guaranteed by Maxwell's equations. Therefore, in the case of $\sigma=0$ at $r=R$, any solution of the induction equation that fulfills the boundary condition $\Psi_{n}=0$ at $r=R$ will be characterized by vanishing tangential surface currents, i.e. $\Psi_{n}=\frac{\partial \Psi_{n}}{\partial r}=0$ at $r=R$.

\subsubsection{Force-free boundary conditions}

The vacuum boundary condition is an idealization. In general, one can also consider the NS surrounded by a magnetosphere in which the internal currents continue. However, its low particle density requires the establishment of a force-free magnetic field configuration above the surface; i.e.

$\nabla \times \boldsymbol{B}=\mu(\boldsymbol{r}) \boldsymbol{B}$,

where $\mu$ can be interpreted as a wavenumber representing the spatial scale of the field in force free conditions, a sort of measure of the thickness of the magnetosphere. The vacuum solution can be recovered by taking the limit $\mu \rightarrow 0$. The divergence-free condition implies $\nabla \mu \cdot \boldsymbol{B}=0$. Assuming that $\mu$ is a constant, and inserting Eqs. (6) and (7) into Eq. (18), it becomes clear that the boundary condition requires

$\Psi_{n}=\mu \Phi_{n}$,

$\frac{\partial^{2} \Phi_{n}}{\partial r^{2}}-\frac{n(n+1)}{r^{2}} \Phi_{n}+\mu^{2} \Phi_{n}=0$.

A variety of possible force-free boundary conditions can be built by varying $\mu$ to test the effect of the size of the magnetosphere on the crustal field evolution. This would give some insight into how the presence of a magnetosphere affects the dissipation efficiency. Naively, one can guess that the dissipation is somewhat less than in the case where vacuum boundary conditions are applied, but still greater than in the purely Ohmic case. The reason is that a force-free boundary condition drives the field to establish itself in a configuration close to force-free at low density, and the effect of the Hall term in the outer crust is reduced.

The main problem with implementing force-free boundary conditions is that the solution will depend on the choice of the function $\mu$, which can be kept fixed to the constant value of an initial configuration or vary with time. The differences due to the variation in the parameter are of the order of the relative difference between vacuum and force-free (vacuum is just the particular case $\mu=0$ ). Since the choice of this function is arbitrary, we restrict ourselves to the external vacuum solution in this paper.

\subsection{Inner boundary conditions}

The inner boundary conditions are determined by the transition from normal to superconducting matter at the crust-core interface $r=R_{i}$. The Meissner-Ochsenfeld effect demands that the normal component of the magnetic field has to vanish at $r=R_{i}$. The continuity of the tangential component of the electric field, together with Ohm's law, forces the component to vanish at $r=R_{i}$, because otherwise the infinite electric conductivity would cause infinite tangential current densities, thereby finally destroying the superconducting state.

For a spherically symmetric NS, the normal component of the magnetic field is its $r$-component as given by Eq. (6). Thus, the inner boundary condition for the poloidal field is

$\Phi_{n}=0$ at $r=R_{i}$

The tangential component of the electric field consists of $\theta$ and $\varphi$-components. For the $\varphi$-component we find, after some algebra:

$$
\begin{aligned}
E_{\varphi}= & \sum_{n, n^{\prime}}\left[\frac{n(n+1)}{r^{3}} \Psi_{n} \frac{\partial \Phi_{n^{\prime}}}{\partial r} Y_{n} \frac{\partial Y_{n^{\prime}}}{\partial \theta}-\frac{n^{\prime}\left(n^{\prime}+1\right)}{r^{3}} \frac{\partial \Psi_{n}}{\partial r} \Phi_{n^{\prime}} \frac{\partial Y_{n}}{\partial \theta} Y_{n^{\prime}}\right] \\
& +\frac{1}{r \omega_{\mathrm{B}} \tau} \sum_{n}\left(\frac{\partial^{2} \Phi_{n}}{\partial r^{2}}-\frac{n(n+1)}{r^{2}} \Phi_{n}\right) \frac{\partial Y_{n}}{\partial \theta} .
\end{aligned}
$$

This electric field component has to vanish at the surface of the superconducting core. Since $\Phi_{n}=0$ at $r=R_{i}$, this condition reads

$\sum_{n, n^{\prime}}\left[n(n+1) \Psi_{n} \frac{\partial \Phi_{n^{\prime}}}{\partial r} Y_{n} \frac{\partial Y_{n^{\prime}}}{\partial \theta}\right]+\frac{r^{2}}{\omega_{\mathrm{B}} \tau} \sum_{n}\left(\frac{\partial^{2} \Phi_{n}}{\partial r^{2}} \frac{\partial Y_{n}}{\partial \theta}\right)=0$

This condition is obviously not suited to finding an inner boundary condition for the toroidal field. Therefore, we have to consider the $\theta$-component of the electric field, which consists of three contributions:

$E_{\theta}=\left[\left(\nabla \times \boldsymbol{B}_{\text {tor }}\right) \times \boldsymbol{B}_{\text {tor }}+\left(\nabla \times \boldsymbol{B}_{\mathrm{pol}}\right) \times \boldsymbol{B}_{\mathrm{pol}}+\frac{1}{\omega_{B} \tau}\left(\nabla \times \boldsymbol{B}_{\text {tor }}\right)\right]_{\theta}$.

Again using the expressions of Eq. (6) and taking into account that $\Phi_{n}\left(r=R_{i}\right)=0$, the term $\left(\nabla \times \boldsymbol{B}_{\mathrm{pol}}\right) \times \boldsymbol{B}_{\mathrm{pol}}$ vanishes, and it remains from the condition $E_{\theta}=0$ at the inner boundary that

$\frac{1}{\omega_{\mathrm{B}} \tau} \sum_{n} \frac{\partial \Psi_{n}}{\partial r} \frac{\partial Y_{n}}{\partial \theta}+\frac{1}{r^{2}} \sum_{n, n^{\prime}} n(n+1) \Psi_{n} \Psi_{n^{\prime}} Y_{n^{\prime}} \frac{\partial Y_{n}}{\partial \theta}=0$

Multiplying both sides with $\frac{\partial Y_{l}^{*}}{\partial \theta}$ and integrating over the solid angle, the orthonormality of the spherical harmonics gives

$\frac{1}{\omega_{\mathrm{B}} \tau} \frac{\partial \Psi_{n}}{\partial r}=-\frac{1}{r^{2}} \sum_{k, k^{\prime}} I^{(2)} \Psi_{k} \Psi_{k^{\prime}}$

Hollerbach \& Rüdiger (2002) applied the above boundary condition in the limit of $\omega_{B} \tau \rightarrow \infty$, thereby avoiding the difficulties the non-linearity will cause. We use the general form of Eq. (25), since during the cooling process of the NS, there certainly will be some periods during which it is not justified to neglect the dissipative term. 


\section{Comments on the evolution of purely toroidal fields}

From Eqs. (4), (9), and (10), it is readily seen that an initial purely toroidal field ( $\Phi=0$ at $t=0)$ will not develop a poloidal part and does remain purely toroidal. For clarity, we will use the $\varphi$-component of the magnetic field $\left(B_{\varphi}\right)$ as a variable in this section. In axial symmetry, it is related to $\Psi$ through

$\boldsymbol{B}_{\text {tor }} \equiv B_{\varphi} \boldsymbol{e}_{\varphi}=-\frac{\partial \Psi}{\partial \theta} \boldsymbol{e}_{\varphi}$

In order to make the effect of the Hall drift evident we use cylindrical coordinates $(R, \varphi, z)$. In Appendix A we write the general form of the induction equation in cylindrical coordinates, with a decomposition of the magnetic field as used by Hollerbach \& Rüdiger (2002). When considering the case of purely toroidal fields, neglecting the dissipative term (limit of strong magnetization) for simplicity, and assuming constant electron density $n_{\mathrm{e}}$, the induction equation is reduced to a single evolution equation for the variable $B_{\varphi}$ :

$\frac{\partial B_{\varphi}}{\partial t}=-\frac{\hat{\tau}}{\hat{\sigma}} \boldsymbol{e}_{\varphi} \cdot \nabla \times\left[\left(\nabla \times \boldsymbol{B}_{\text {tor }}\right) \times \boldsymbol{B}_{\text {tor }}\right]=\frac{c}{4 \pi e n_{\mathrm{e}}} \frac{2 B_{\varphi}}{R} \frac{\partial B_{\varphi}}{\partial z}$,

where we have used $\hat{\tau} / \hat{\sigma}=c / 4 \pi e n_{\mathrm{e}}$.

In this form, the Hall-dominated induction equation has the form of the inviscid Burgers equation with a wave velocity that depends on the variable $B_{\varphi}$ and on the coordinate $R$. This has been pointed out by Reisennegger et al. (2005), who also conclude that it leads to the formation of current sheets. In this way, the formulation illustrates very clearly that the originally (in the limit of weak field) purely parabolic diffusion equation changes its character to hyperbolic when the Hall term dominates. The analogy between the induction equation and the Burgers equation has been discussed before by Vainshtein et al. (2000), but there are a few more qualitative differences that we should notice. Vainshtein et al. (2000) derived the analogy with the Burgers equation in Cartesian coordinates, assuming no dependence of the field on the $z$-coordinate. In this particular case, it is necessary to consider a stratified medium in order to have the Hall drift lead to the formation of current sheets. Our result is more general: even in the uniform density case, the geometry of a NS crust (axially symmetric toroidal field in a conducting spherical shell) leads to a Burgers-like equation that does not admit stationary solutions. Depending on the sign of the gradient, the field will drift vertically in one or the other direction, leading to the formation of current sheets either at the surface or at the crust-core interface. The purely vertical drift acting within a spherical shell (the crust) will inevitably result in fast dissipation locally, wherever current sheets are formed. Moreover, when we consider NS models with a stratified electron number density, the gradient in $n_{\mathrm{e}}$ through the NS crust may additionally support the creation of current sheets (mainly close to the crust-core interface).

But there is yet another important problem related to the change from the parabolic to hyperbolic character of the induction equation for purely toroidal fields. It is well known that the solutions of the inviscid Burgers equation develop discontinuities or, if a small viscous term is present, steep gradients. This is probably the reason spectral methods have always failed when trying to numerically solve the induction equation for large magnetization parameters. The system evolves naturally to form a discontinuity or sharp gradient in $B_{\varphi}$ on a characteristic timescale that can be shown to be $\approx \tau_{\text {Hall }}$ (the Hall timescale is defined below in Eq. (32))

$\tau_{\text {shock }}=\left(\frac{c}{2 \pi e n_{\mathrm{e}} R} \frac{\partial B_{\varphi}}{\partial z}\right)^{-1}=\frac{R \hat{\sigma}}{2 \hat{\tau}}\left(\frac{\partial B_{\varphi}}{\partial z}\right)^{-1}$,

which is on the order of $\approx 10^{6}-10^{7} \mathrm{yr}$ for $B=10^{13} \mathrm{G}$ and a typical lengthscale of $1 \mathrm{~km}$. However, it can be much shorter for stronger fields and small-scale structures generated by the Hall cascade. A similar timescale characterizes the typical travel time of a magnetic perturbation to reach either the surface or the corecrust boundary,

$\tau_{\text {travel }}=\left(\frac{c}{2 \pi e n_{\mathrm{e}} R} \frac{B_{\varphi}}{d}\right)^{-1}$

where $d$ denotes the distance between the initial position of the perturbation in the crust and the surface or the crust-core interface. The formation of a shock by the compression of the field against the inner or outer boundaries will cause unavoidable numerical instabilities and/or the Gibb's phenomenon, if one tries to solve the problem by means of spectral methods. Note that, locally and temporarily, the three characteristic timescales $\left(\tau_{\text {Hall }}, \tau_{\text {shock }}\right.$, and $\left.\tau_{\text {travel }}\right)$ may coincide or differ by orders of magnitude, because they are proportional to the second, first, and zeroth derivative of the field strength, respectively. These characteristic timescales become different once small-scale structure or current sheets appear. This makes any reasoning based on those timescales questionable; only a serious numerical study of the Hall drift can yield an idea as to its effects on the crustal magnetic field evolution.

For the simple case of a Burgers-like equation with constant $n_{\mathrm{e}}$, (see Eq. (27)), we have checked that upwind methods, specifically designed to deal with hyperbolic equations, work very well in regimes $\omega_{B} \tau \rightarrow \infty$, in which a spectral method fails. Thus, the numerical problems observed with spectral codes for $\omega_{B} \tau \gtrsim 100$ are most likely caused by an intrinsic limitation of the numerical approach. In the general case, having both poloidal and toroidal field components in a stratified spherical shell, the field evolution is not so simple. Then, the equations are strongly coupled, they have both a parabolic and a hyperbolic part, and it is difficult to guess what the best strategy is for solving them. It is not the scope of this paper to give the final answer about the best numerical technique. We intend to point out in this section that there are deep unavoidable reasons that lead to unsurmountable problems in many cases. For the rest of the paper, we focus on simulations with realistic NS models that can be handled by our hybrid method. We take advantage of a rare occasion: the more realistic model causes less numerical problems than the constant density model. The reason is that current sheets are smoothed out more quickly in the shell layers just below the surface, because their electric conductivity is orders of magnitudes lower than in the inner crust. In addition, in the long run, the toroidal field seems to find a quasi-equilibrium configuration in which the effect of the gradient of conductivity counterbalances the Hall drift. Such an equilibrium can not be established for non-stratified crustal models.

\section{The NS model and the initial conditions.}

Our aim is to study the global evolution of the magnetic field in isolated NSs. The Hall drift occurs both in the fluid core and in the solidified crust. While the effect of the Hall drift in the core is less obvious and may proceed on a timescale on the order of 
the Hubble time (insert $L_{5}=10$ and $\rho \approx 10 \rho_{\text {nuc }}$ into Eq. (61) of Goldreich \& Reisenegger 1992), its effect can be crucial in the crustal field.

To build the background NS model we have used a modern Skyrme-type equation of state (EOS) at zero temperature, describing both the NS crust and the liquid core, based on the effective nuclear interaction SLy (Douchin \& Haensel 1991). The low-density EOS (below the neutron drip point) employed is that of Baym et al. (1971). With this EOS, we built a NS model with a radius of about $11.7 \mathrm{~km}$ and a mass of $1.28 M_{\odot}$. The central density is $8.83 \times 10^{14} \mathrm{~g} / \mathrm{cm}^{3}$, and its crust (from $\rho \approx 10^{10} \mathrm{~g} / \mathrm{cm}^{3}$ to $\approx 10^{14} \mathrm{~g} / \mathrm{cm}^{3}$ ) extends from 10.7 to $11.6 \mathrm{~km}$. In Fig. 1 we show radial profiles of the electric conductivity $\sigma$ and $\omega_{B} \tau / B_{13}$, where $B_{13}$ is $B$ in units of $10^{13} \mathrm{G}$ for three different temperatures. We assumed a constant impurity concentration parameter $Q=n_{\text {imp }}\left(Z_{\text {imp }}-Z\right)^{2} / n_{i}$ of $Q=10^{-2}$. Here, $n_{\text {imp }}$ and $n_{i}$ are the impurity and ion particle densities, respectively, and $Z_{\text {imp }}$ is the charge number of the impurities. We compared simulations with $Q=10^{-2}$ and $Q=10^{-4}$ without finding significant differences. A high impurity content could lead to even faster dissipation (Jones 2004). The figure demonstrates how the electric conductivity varies by 3-4 orders of magnitude within the crust and depends strongly on the temperature. The magnetization parameter scales linearly with $B$. For the fiducial field of $10^{13} \mathrm{G}$, it is of the order of unity for a temperature of $10^{9} \mathrm{~K}$ but can become as large as 1000 as the star cools down. For magnetar field strength which both at the surface (radial poloidal component) and within the crust (meridional poloidal and toroidal components) may well exceed $B_{13}=10$, the magnetization parameter can locally reach values in excess of $10^{4}$.

From Fig. 1 one can also read the relevant timescales of the problem. The Ohmic timescale is

$\tau_{\mathrm{Ohm}}=\frac{4 \pi \sigma \lambda^{2}}{c^{2}}=\hat{\sigma} \lambda^{2}$

where $\lambda$ is the typical magnetic field length-scale. Inserting some typical numbers, we get

$\tau_{\mathrm{Ohm}}=4.4\left(\frac{\sigma}{10^{24} \mathrm{~s}^{-1}}\right)\left(\frac{\lambda}{\mathrm{km}}\right)^{2} 10^{6}$ years.

On the other hand, the Hall timescale is

$\tau_{\text {Hall }}=\frac{4 \pi e n_{\mathrm{e}} \lambda^{2}}{c B}$.

The ratio of the Ohmic to Hall timescale is simply given by $\omega_{B} \tau$. From Fig. 1 we can infer that, if the temperature of a NS's crust varies between $10^{9}$ and $10^{8} \mathrm{~K}$ during the first million years of its life, the average Ohmic timescale in the crust is $\approx 1 \mathrm{Myr}$. For magnetized NSs, the ratio of the Ohmic to Hall timescale is approximately

$\frac{\tau_{\text {Ohm }}}{\tau_{\text {Hall }}}=(1-10) \times B_{13}$.

Note, however, that such averaged timescales are of very restricted use in characterizing the field evolution in NS crusts, since both the density and the magnetic field vary over many orders of magnitude there. There is no doubt, then, that in any circumstances there is magnetic field decay in the crust of young NSs. A different issue is whether or not this effect is observable when studying populations of older NSs. Because of the strong temperature dependence of the electrical conductivity, when a NS's crust cools below $10^{7} \mathrm{~K}$, typically one million years

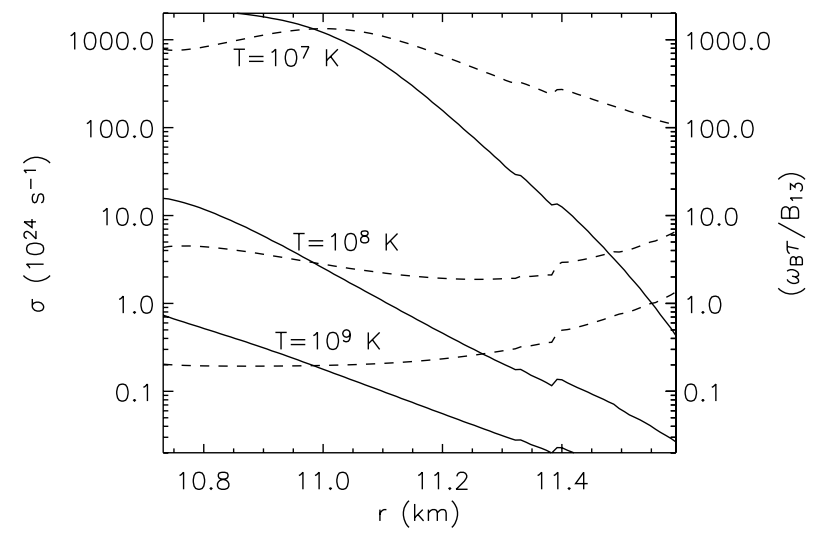

Fig. 1. Radial profiles of electrical conductivity $\sigma$ (solid lines), and the magnetization parameter normalized to a fiducial magnetic field of $B_{13}=10^{13} \mathrm{G}$ (dashed lines), for three different temperatures. The crust is assumed to be isothermal.

after birth, the Ohmic dissipation time increases significantly, and no rapid field decay can be expected after that age.

For this reason, we want to focus in this paper on the initial evolution of magnetic fields in relatively young NS. In order to mimic the cooling process of a NS, we started with a crust at $T=$ $10^{9} \mathrm{~K}$, a typical value after formation of the crust, at most within hours after birth, and we force the temperature of the isothermal crust to vary according to

$T(t)=10^{9}\left(1+10^{6} t_{6}\right)^{-1 / 6}$

where $t_{6}$ is the NS age in $10^{6}$ years. This approximation is valid during the neutrino-cooling era if only modified URCA processes are operating (Page et al. 2006). It is not the purpose of this paper to discuss how the thermal and magnetic field evolutions are coupled in detail, but this simple approximation is sufficient for capturing the main effect: as the NS's crust cools (from $10^{9}$ to $10^{8} \mathrm{~K}$ in about $1 \mathrm{Myr}$ ), via the increase in the electron relaxation time $(\tau)$, the Hall term becomes more and more important. When a significant part of the crustal field is dissipated and/or it has approached a force-free configuration, its decay continues on a much longer Ohmic timescale. We have not included effects of temperature anisotropies within the crust, although they may be important, but it will be addressed in detail in future works.

The other microphysical input needed for performing the simulations is the electrical conductivity and the magnetization parameter. Since the magnetization parameter and the electrical conductivities vary as the temperature drops and the magnetic field evolves, we recompute the value of the relaxation time and the electrical conductivities at each time step by using the public code developed by A. Potekhin (1999) ${ }^{2}$. Despite its causing some numerical efforts, we have chosen to be as realistic as possible and use this state-of-the-art microphysical ingredients to account for the effect of composition stratifications.

\subsection{Initial magnetic field configuration}

Little is known with certainty about the initial magnetic field strength and structure. Recently, Braithwaite (2006) and Geppert \& Rheinhardt (2006) have shown that sufficiently rapid rotation can stabilize dipolar toroidal and poloidal fields of magnetar strength against MHD instabilities occurring immediately

\footnotetext{
${ }^{2}$ wwW.ioffe.rssi.ru/astro/conduct/condmag.html
} 
Table 1. Description of initial models, differing in the initial field strength and relative sign of the poloidal and toroidal components. All initial poloidal fields are purely dipolar $(n=1)$.

\begin{tabular}{cccc}
\hline \hline Model & $\begin{array}{c}B_{\text {pol }} \\
\left(10^{13} \mathrm{G}\right)\end{array}$ & $\begin{array}{c}B_{\text {tor }} \\
\left(10^{13} \mathrm{G}\right)\end{array}$ & $\begin{array}{c}\text { Multipole } \\
\text { (toroidal) }\end{array}$ \\
\hline $\mathrm{A}$ & 10 & -100 & $n=2$ \\
$\mathrm{~B}$ & 10 & 100 & $n=2$ \\
$\mathrm{C}$ & 10 & -100 & $n=1$ \\
$\mathrm{D}$ & 1 & -10 & $n=2$ \\
$\mathrm{E}$ & 20 & -200 & $n=2$ \\
$\mathrm{~F}$ & 50 & -50 & $n=1$ \\
$\mathrm{G}$ & 100 & -100 & $n=2$ \\
$\mathrm{H}$ & 10 & -100 & $n=1(\mathrm{FF})$ \\
\hline
\end{tabular}

after completion of the proto-NS phase. Only preliminary results are available on the stability of magnetic field structures consisting of a poloidal-toroidal mixture. Our conclusion about the origin of strong toroidal crustal fields that probably exist in magnetars and quite certainly exist in thermally emitting NSs with a highly non-uniform surface temperature distribution (Geppert et al. 2004, 2006; Pérez-Azorín et al. 2006a,b) is, therefore, that they have probably been generated at birth. Later, they were stabilized against Tayler instabilities and have been frozen into the solid crust when it forms. Alternatively, the presence of sufficiently strong temperature gradients both close to the crustcore interface and in the degenerate surface layers (Wiebicke \& Geppert 1995) are able to convert thermal into magnetic energy very effectively via a thermoelectric instability. In this case, the magnetic energy will be stored predominantly in toroidal field structures.

We have considered quite different initial structures: purely poloidal and purely toroidal ones, with initial dipolar and quadrupolar modes, and several mixed initial fields where the ratio between the energies stored in the toroidal and poloidal field parts is varied. For the poloidal field, our initial configurations are chosen to be free Ohmic decaying modes (strictly speaking, pure decay modes for constant conductivity profiles) satisfying the boundary conditions. For a given angular multipole $(n)$, the radial eigenfunctions can be written as linear combination of spherical Bessel functions of the first $\left(\mathrm{j}_{n}\right)$ and second $\left(\mathrm{n}_{n}\right)$ kind

$\Phi_{n}(r)=a r \mathrm{j}_{n}(\mu r)+b r \mathrm{n}_{n}(\mu r)$

where $\mu$ is the radial wavenumber. The boundary conditions fix the wavenumber $\mu$ and the ratio $b / a$.

For our crust model, we have $\mu=1.8353 \mathrm{~km}^{-1}, a=1$, and $b=\tan (\mu R)$. For the models presented in this paper, the overall normalization factor has been chosen to fix the radial component at the pole of the dipolar $(n=1)$ component to the values of $B_{\mathrm{pol}}$ listed in Table 1.

In Table 1 we summarize the models employed in this work, differing in the initial values and relative sign of the poloidal and toroidal components. For the Models A to G, the initial toroidal field is given by

$\Psi_{n}(r)=c\left[\left(r-R_{i}\right)(r-R)\right]^{2}$

where $c$ is a constant determined by the maximum value of $B_{\varphi}$. These configurations are initially non-force-free, and thus exert Lorentz forces on the crust. They were chosen after exploring different initial configurations, because they were found to evolve more smoothly, minimizing the effects of the initial imbalance. For comparison, we also include model $\mathrm{H}$, which is a force-free configuration satisfying

$\Psi=\mu \Phi(r)$.
In a constant conductivity plasma, and neglecting the effects of Hall drift (a force-free field is not subject to it), this is an eigenfunction of the induction equation. However, due to the gradient of conductivity, the force balance is immediately broken at the beginning of the evolution, and the Hall term is activated.

\section{Results and discussion}

We present the results now of our numerical simulations of the field evolution in the crust and its dependence on the initial magnetic field structure and strength. We restrict this presentation to the selection of initial models described in Table 1, although we have performed a number of different simulations with a variety of initial configurations. In particular, we reproduced some of the toy models (constant $\hat{\sigma}$ and $\hat{\tau}$ ) found in the literature (Hollerbach $\&$ Rüdiger 2002) to test our code. We saw a good qualitative agreement with only minor quantitative differences. We also performed simulations of the evolution of purely toroidal fields, which remain toroidal forever, for testing purposes. In particular, we checked that the evolution of a purely toroidal field, in the limit of very low resistivity and with constant $n_{\mathrm{e}}$ evolves according to Eq. (27), i.e., it is only subject to a vertical drift with a velocity that depends on the distance to the axis and the field intensity. We also tested the diffusive part of the code (by setting $\hat{\tau}=0$ and $\hat{\sigma}=$ constant) against the analytical solutions in terms of spherical Bessel functions. We found that the analytical and numerical solutions differ in less than $0.2 \%$ for a typical resolution of 100 radial shells. For conciseness, in this section we only discuss realistic models of magnetized NSs.

In Figs. 2-4 we present the evolution of models A, B and $\mathrm{C}$. For three representative ages, we show both the poloidal magnetic field lines (top panels) and the contours of constant toroidal field strength (bottom panels). The crustal shell has been stretched by a factor of 4 for clarity. Models A and B differ only in the relative sign of its toroidal component, which for both models is initially quadrupolar $(n=2)$, while model $\mathrm{C}$ has an initially dipolar $(n=1)$ toroidal field with the same radial profile as model A. In all cases the models have the same maximum toroidal field strength. The different sign of the toroidal field affects the direction of the Hall drift (toward north or south). The other important effect is due to the gradient of the electric conductivity, which always causes the tendency to displace field lines towards the inner crust.

For model A, we see in the central panel how the Hall drift compresses magnetic field lines near the equator, while the results for model B show the opposite tendency. Due to its dominant initial dipolar structure, model $\mathrm{C}$ shows a global displacement of the magnetic field towards the south pole, which results in a fast dissipation in the high resistivity surface region near the south pole. During that phase, the interplay of the poloidal and toroidal field modes is very intense. The magnetic field is dragged and twisted, thereby creating current sheets. At these sites of very efficient dissipation, the toroidal field weakens rapidly and, after becoming almost as strong as the poloidal field, the latter tends to return to its original position. This sequence of twisting and stretching and subsequent release of the twisted field characterizes this epoch by its oscillatory behavior. However, looking at the right panels, we see that, after about half a million years, all poloidal fields have a very similar appearance. The strongly dissipated, and now weaker, toroidal 

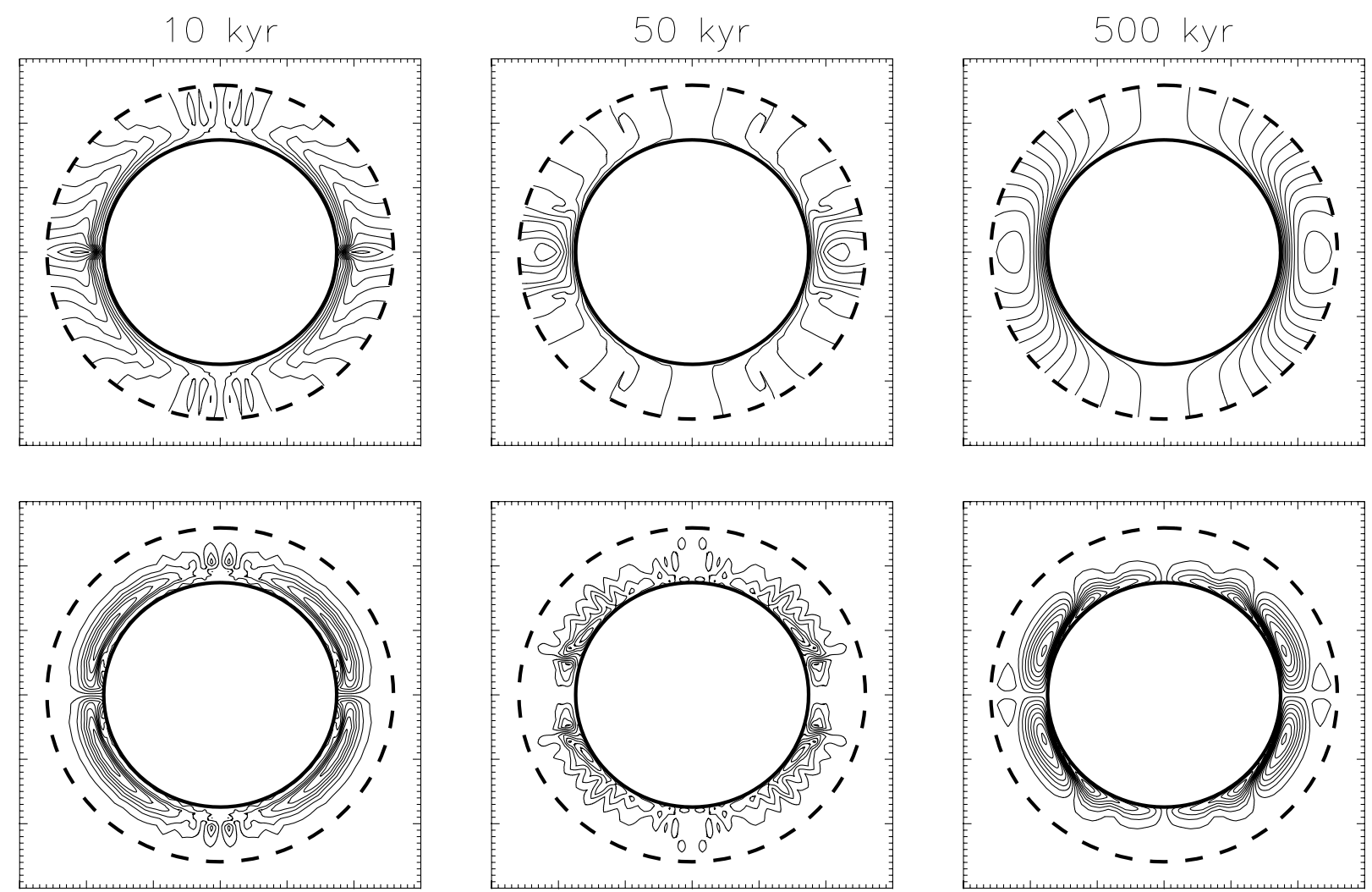

Fig. 2. Top: poloidal magnetic field lines for three different evolutionary stages of the magnetic field configuration initially described by model A. $(t=10,50$, and $500 \mathrm{kyr})$. Bottom: contours of constant toroidal field strength $\left(B_{\varphi}\right)$. The crustal shell has been stretched a factor of 4 for clarity.

components, become more regular than in the Hall phase, and they are dominated by the $n=2$ or $n=3$ modes $^{3}$.

The main conclusion from these results is that the initial magnetic field configuration largely determines the early evolution $\left(<10^{5} \mathrm{yr}\right)$. In the long run, however, there seems to exist a clear tendency to establish a more stable configuration, consisting of a dipolar poloidal component in combination with higherorder toroidal field modes. This fact can be better understood when looking at the evolution of the radial profiles of the dominant modes, as shown in Fig. 5. At early times, say $t=20 \mathrm{kyr}$, the interplay between the toroidal and poloidal components results in a different evolution of $\Phi_{1}$, although the shape is qualitatively similar. After half a million years, the poloidal field is clearly dominant and very similar for all models. The toroidal field is weaker (in real units, about a factor of 2-3 smaller than the poloidal field) and concentrated towards the inner crust. It is interesting to notice that $\Psi_{2}$ is also rapidly driven towards a similar shape in all cases, indicating that after the fast initial transient the gradient of the conductivity determines a sort of quasi-equilibrium field.

It is also interesting to look at the evolution of the total magnetic energy (Fig. 6) to understand the different evolutionary stages that the NS passes through. First, it is remarkable how all models have a very similar evolution and how they converge at late times towards a similar exponential decay. For all models, the magnetic energy has been dissipated by a factor 10 to 20

\footnotetext{
3 The black/white figures presented here are snapshots and yield only a vague impression of the evolution of the magnetic field with Hall drift and Ohmic diffusion, everything coupled with the cooling of the crust. We advise the reader to look at the movies, available at http://www.dfa.ua.es/UNS06/movies.html, where the evolution is visualized in much more detail.
}

after 0.5 Myr. Second, we can clearly differentiate between the initial fast decay, in which the field can dissipate one half of its energy in only $\sim 10^{4} \mathrm{yr}$. This is caused by the much lower initial conductivity at higher temperatures combined with the effects of the Hall drift. During the subsequent stage, the Ohmic dissipation timescale (about half a million years) is almost identical for all models. Obviously, the first stage is very important for models with initially stronger fields, i.e. it will play an important role for NSs born as magnetars. This seems to be the only period, before the photon cooling era, when the Hall drift will qualitatively affect the field evolution. In general, the models in which the initial ratio of toroidal to poloidal field is small (models F, G) decay slower than those with large ratios (models A, B, C, E). Among the latter, the stronger the initial field, the more efficient is the dissipation during the Hall phase.

The comparison of models $\mathrm{C}$ and $\mathrm{H}$ is also interesting. The have a similar amount of magnetic energy, the only difference being that model $\mathrm{H}$ is initially force-free but model $\mathrm{C}$ is not. This makes it clear that the early part of the evolution due to the initial imbalance is very short (a few kyrs). The gradient of conductivity is what determines the long-term quasi-stationary configurations (as shown in Fig. 5). Our conclusion after exploring many more models not shown in this paper is that the initial field structure is rapidly reorganized and that the conductive properties of the crust determine the field configuration in NSs with ages $>10^{4} \mathrm{kyr}$.

The Ohmic dissipation rate appears to be faster than what has been usually discussed in the literature. The reason is that we are simulating the evolution of NS models for the first time with a realistic profile of electron density, composition, conductivity, etc., including the coupling between different field modes through the non-linear Hall term. The effect of the Hall drift is 

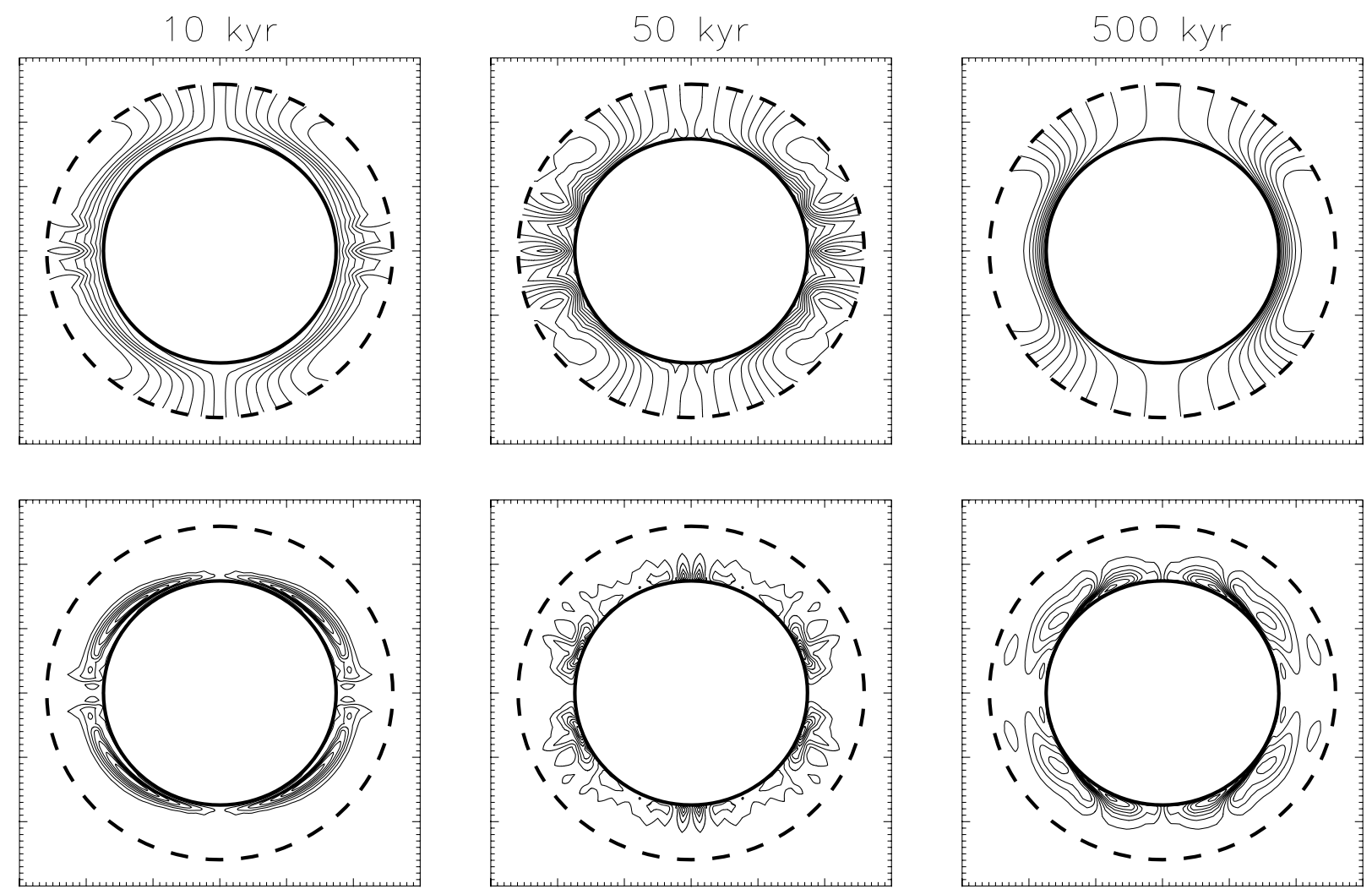

Fig. 3. Same as Fig. 2 but for model B.

twofold: it causes the transfer of magnetic energy to small-scale modes that decay faster and, in some cases, the displacement of the magnetic field to regions of higher resistivity where it is rapidly dissipated.

In Fig. 7 we compare the evolution of the total magnetic energy in models $A$ and $G$ with the hypothetical evolution of the same initial configuration without the Hall drift (by setting $\hat{\tau}=0$ ). After the initial phase (about $50 \mathrm{kyr}$ ), during which the models that include the Hall drift dissipate energy about $10-20 \%$ faster, the rate of energy dissipation becomes approximately the same. This shows that the initial departure of the exponential decay is partially caused by Hall drift, but also by the relatively rapid cooling of the crust that results in a significant time-dependence of the conductivity and of the magnetization parameter. Other cooling models, for example fast cooling due to the activation of direct URCA processes, may result in an initially different evolution. However, we think that, on a secular timescale of about $0.1 \mathrm{Myr}$, the system readjusts itself and the dissipation of magnetic field is mostly controlled by the Ohmic decay. In a future work we plan to couple a multidimensional cooling code with the magnetic field evolution to study different cooling scenarios.

To finish the discussion in this section, we present in Fig. 8 the power spectra for models $\mathrm{A}, \mathrm{B}$, and $\mathrm{C}$ at three different moments of the evolution. In the left panel, which corresponds to $t=1 \mathrm{kyr}$, we see how the Hall cascade is filling the shorter wavelength modes very quickly (initially only $n=1$ and $n=2$ modes existed). At $t=10 \mathrm{kyr}$, which corresponds roughly to the Hall timescale, the Hall cascade and perhaps the Hall instability have filled out all large wavenumber modes and approximately saturates following a $n^{-2}$ power law. This situation is kept for about $50 \mathrm{kyr}$ (not shown in the figure), until the field has been dissipated by a significant amount, and the Hall term has begun to lose its importance with respect to the regular Ohmic dissipation term. After half a million years, the power spectrum is much steeper $\left(\propto n^{-6}\right)$, an indication that the Hall drift has lost its influence on the crustal field evolution.

\subsection{Spin-down evolution}

Since the evolving crustal field may also have effects on the rotational evolution of the NS, we study the differences in the spin-down behavior between different models. In Fig. 9 we show the evolution of the period (top), period derivative (middle), and spin-down age $\left(t_{\mathrm{sd}}=P /(2 \dot{P})\right)$ (bottom) in four selected models with different initial dipolar field strengths. The line-styles correspond to models A, D, E, and G. A typical NS born as a magnetar would correspond to model G or, perhaps, E, while a typical pulsar such as Geminga would show the behavior of model D.

Notice that a minimum initial poloidal field of about $2 \times$ $10^{14} \mathrm{G}$ is required to explain the large rotation periods (5-10 s) of thermally emitting, isolated NSs such as the Magnificent Seven. This may be another indication of a common evolutionary path in which some INSs were born as magnetars and their magnetic field has decreased one order of magnitude during their lifetimes. The evidence of crustal field decay presented here also has implications for estimates of the ages of old NSs. Pulsar spin down is thought to generally follow the prediction of the vacuum dipole model, which gives $\dot{v} \propto B^{2} v^{3}$, where $v$ is the spin rate. If the birth spin rate far exceeds the present spin rate and $B$ is constant, the age in this model is given by $t_{\mathrm{sd}}$. This expression is used as the standard estimate of a pulsar's age. If the field is decaying according to our simulations, however, the relationship between the true age and $t_{\mathrm{sd}}$ given by the dipole model is shown in the bottom panel of Fig. 9. It shows that $t_{\mathrm{sd}}$ seriously overestimates the age for NS older than $10^{5}$ years. This effect helps to 

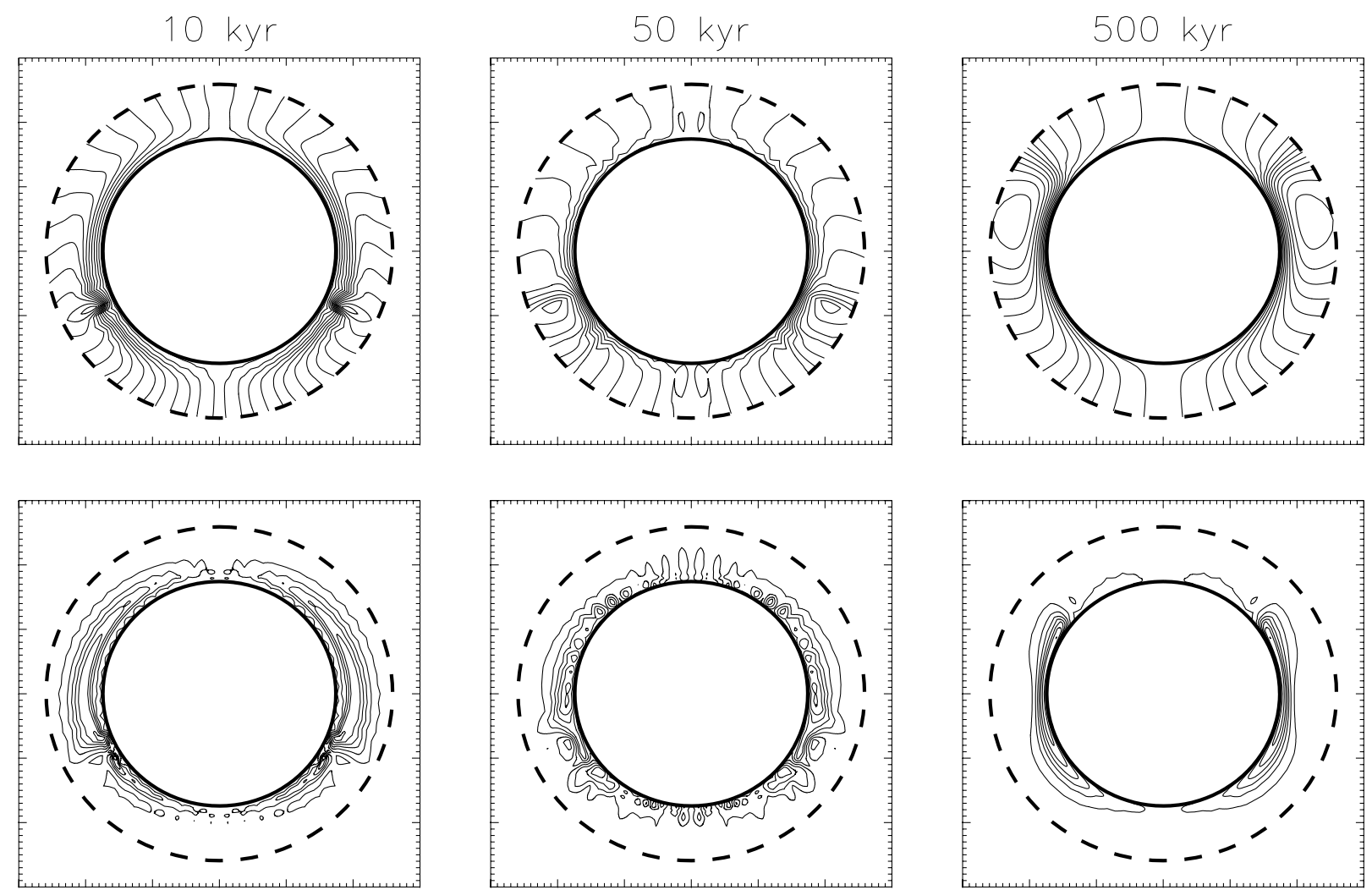

Fig. 4. Same as Fig. 2 but for model C.

reconcile the observed discrepancy between the spin-down ages and independent measures of the ages of some isolated NSs.

Another interesting feature is the rapid variation in the dipolar poloidal component during the Hall epoch. Of course, the total magnetic energy always decreases, but the $n=1$ poloidal component may exhibit an oscillatory behavior. This is a result of the efficient energy transfer between the large-scale toroidal and the dipolar poloidal field modes, i.e. a genuine effect of the Hall drift. Assuming that only the $n=1$ component contributes to spin the star down, the oscillations of the dipolar poloidal surface field affect the braking index $n=v \ddot{v} / \dot{v}^{2}$, as shown in Fig. 10. During the first $\sim 10^{5}$ years of their lives, sufficiently magnetized NSs will show a very erratic variation of the braking index, which may reach any value from 1.5 to 4 . The oscillations of the braking index continue even until $\sim 5 \times 10^{5}$ years, when the initial toroidal field is stronger, as seen for model $\mathrm{E}$ that has an initial maximum toroidal field of $2 \times 10^{15} \mathrm{G}$. At late times, once Ohmic dissipation is controlling the evolution and the dipolar magnetic field decreases steadily, the braking index gradually increases and might reach very high values. This process will be inverted after the crustal field is dissipated almost completely and the rotational evolution of the NS is determined by the much longer timescale of the core field expulsion. In that period, the braking index will approach its canonical (dipolar) value of 3 again. Our results for the braking index evolution coincide very well with the observed indices for middle-aged pulsars (see Geppert \& Rheinhardt 2002). It can be shown that the braking index satisfies the relation

$n=3-2 \frac{\dot{B} P}{B \dot{P}} \approx 3+4 \frac{t_{\mathrm{sd}}}{\tau_{B}}$,

where we have introduced a typical magnetic field decay time $\tau_{B}=B / \dot{B}$ to derive the latter equality. The thin solid line in
Fig. 10 corresponds to this approximation with $\tau_{B}=0.8 \mathrm{Myr}$. Notice that Fig. 7 shows the evolution of the total magnetic en$\operatorname{ergy}\left(\propto B^{2}\right)$, so that the decay time for the magnetic field is twice the value quoted in Fig. 7.

It should be noted that neutrino emission no longer controls the thermal evolution of NSs after $\approx 1 \mathrm{Myr}$, so our results do not apply. Moreover, the low temperatures reached at that time would increase the conductivity significantly (see Fig. 1). Then, the purely Ohmic decay will proceed more slowly, but also the magnetization parameter will increase, which may result in another Hall stage during the photon cooling era. In future work, we plan to extend our study to longer times with consistent temperature evolution. In addition, one always must keep in mind that the magnetic field component supported by currents in the superconducting NS core would be dominant after the crustal field has been dissipated. This, however, is a completely different scenario that cannot be analyzed with our presently available tools.

\subsection{The Hall instability?}

We have seen in our numerical studies some hints of the occurrence of the Hall instability, i.e., of the rapid, non-local in the momentum space, energy transfer from the initial large scale field modes into much smaller ones. Related spectral features can be seen e.g. in Fig. 8, where local maxima appear. However, at the present level of energy conservation (99.9\%) and of our limiting angular resolution $\left(n_{\max }=120\right.$, although most of our runs were performed with $n_{\max }=50$ ), we cannot distinguish the truncation effects from the onset of the Hall instability. Therefore, we decided to postpone the study of the Hall instability in the NS crust to future work. 

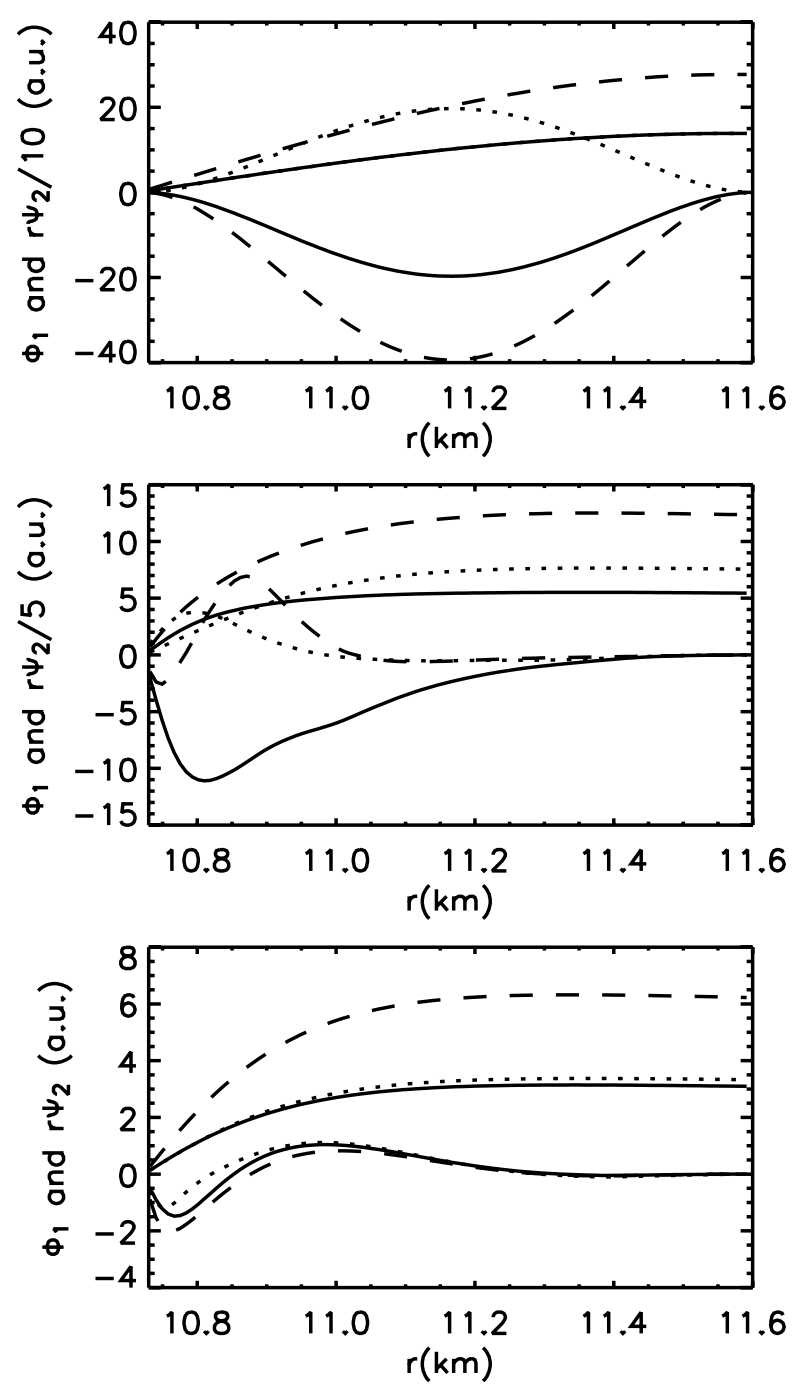

Fig. 5. Radial profiles of $\Phi_{1}$ and $r \Psi_{2}$ (in arbitrary units). These quantities are proportional to the poloidal (dipolar) and toroidal (quadrupolar) components, respectively (see Eq. (6)). The upper panel shows the initial model, while the central and bottom panels correspond to $t=20 \mathrm{kyr}$ and $t=500 \mathrm{kyr}$, respectively. The different line styles stand for models A (solid line), B (dotted line), and E (dashed line). Notice the different normalization of $r \Psi_{2}$ in the upper and middle panels (a factor of 10 and 5 , respectively).

\section{Conclusions}

The results presented above show that magnetic fields maintained by currents circulating in the crust of NSs are strongly rearranged and do decay significantly during the first million years of a NS's life. In addition to purely Ohmic decay, which is faster in the first thousands of years when the electrical conductivity is relatively low due to the high crustal temperature, we find that the Hall drift may contribute noticeably to accelerating the dissipation of magnetic fields. For typical field strengths of $10^{14} \mathrm{G}$, we observe a stage dominated by Hall drift followed by purely Ohmic decay proceeding on a timescale on the order of 1 Myr. Depending on the strength and structure of the initial magnetic field, this Hall phase lasts a few $10^{3}-10^{4}$ years and is characterized by an intense exchange of magnetic energy between the poloidal and toroidal components of the field and by the redistribution of magnetic field energy between different scales. It can be expected that such rearrangements and the relatively rapid

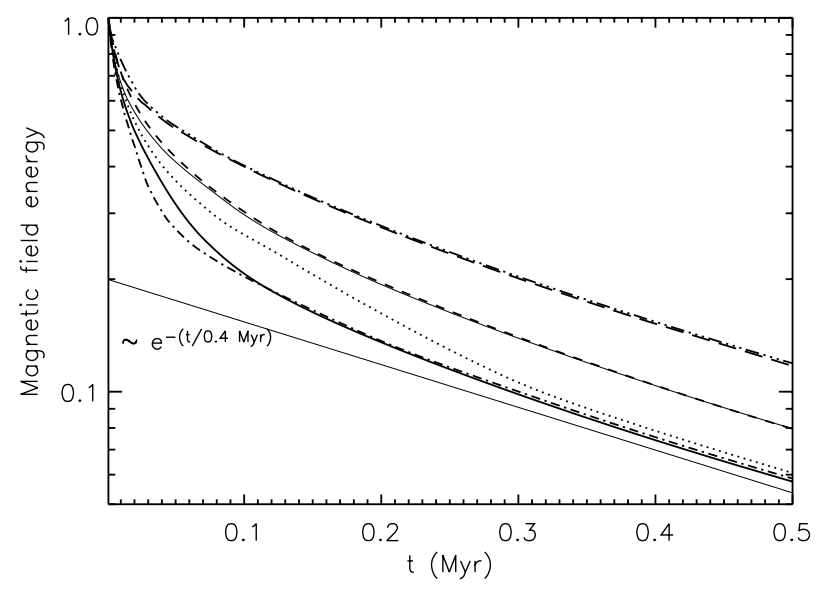

Fig. 6. Evolution of the total energy stored in the magnetic field, normalized to the initial value, for models A (thick solid), B (dotted), C (short dashes), E (dot-dash), F (triple dot-dash), G (long dashes), and $\mathrm{H}$ (thin solid). The straight line shows, for comparison, an exponential decay with a timescale of $\approx 0.4 \mathrm{Myr}$.

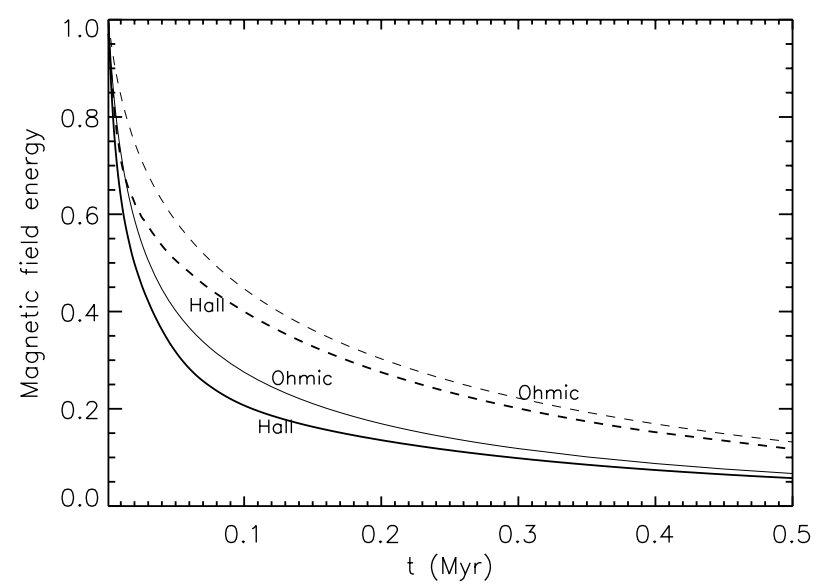

Fig. 7. Evolution of the total energy stored in the magnetic field for models A (solid) and G (dashed) compared with the same initial configurations evolving only by Ohmic decay (thin lines). The difference between thick and thin lines is due to the nonlinear Hall terms that result in a faster initial dissipation.

field decay have observational consequences, as can be observed in magnetars. Of course, whether this first phase plays a large role, and how distinctive it is depends on the initial field strength and structure and how fast the NS cools. If a NS begins its life as a magnetar, its external dipolar field is $>10^{14} \mathrm{G}$. Within the crust, however, the internal magnetic field may locally exceed that value by about one order of magnitude. Thus, even the expected initial high crustal temperatures of $\gtrsim 10^{9} \mathrm{~K}$, which cause a relatively small electron relaxation time $(\tau)$, cannot avoid that $\omega_{B} \tau \gg 1$ in a large fraction of the crust volume.

The toroidal part of the field is specially affected by the Hall drift. There are two main effects acting upon the toroidal fields: it is globally displaced toward the inner crust because of the negative conductivity gradient and, depending on the relative sign with respect to the poloidal component, it tends to move vertically toward one or the other magnetic pole. After the Hall stage, during which the toroidal field is strongly rearranged and dissipated, the long-term evolution seems to select, generally, a predominantly quadrupolar/octupolar structure concentrated in the inner crust and with tendency to be stronger close to the poles. This multipolar structure will determine the surface temperature 

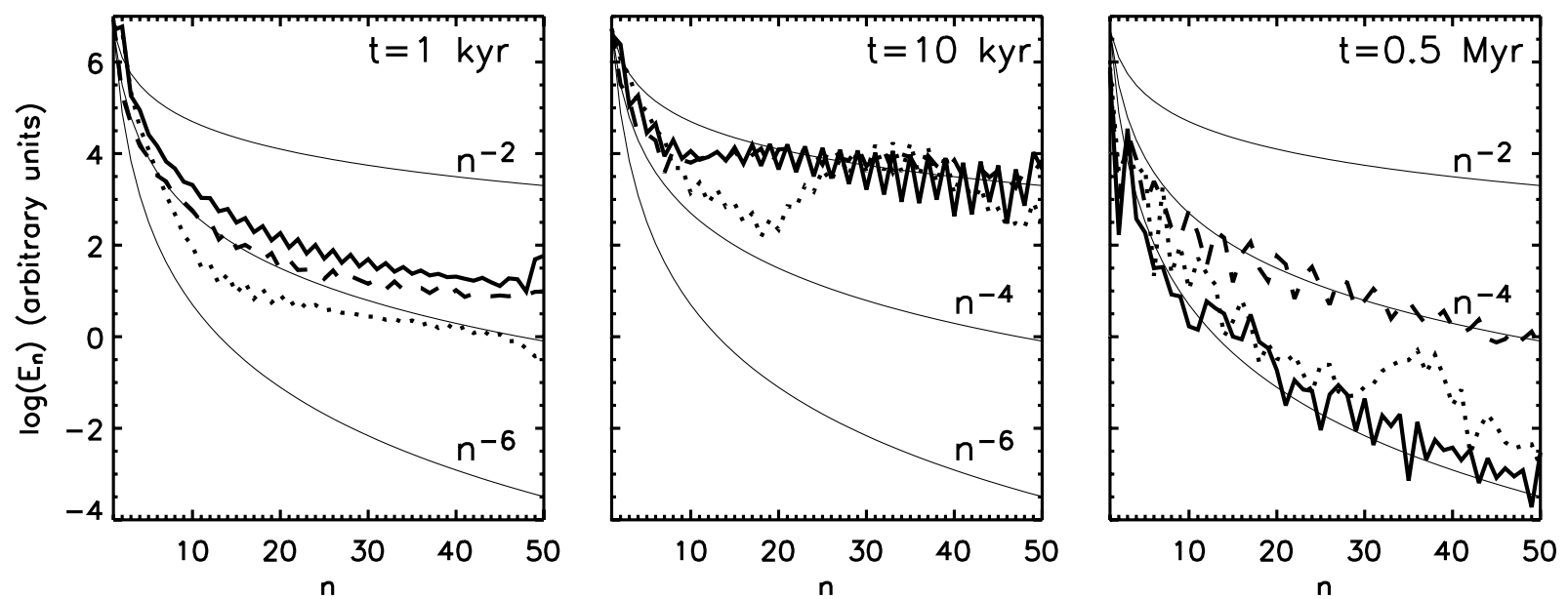

Fig. 8. Power spectrum for models A (solid lines), B (dots), and C (dashes) at three different epochs. For comparison, we also show three different power laws (thin lines).
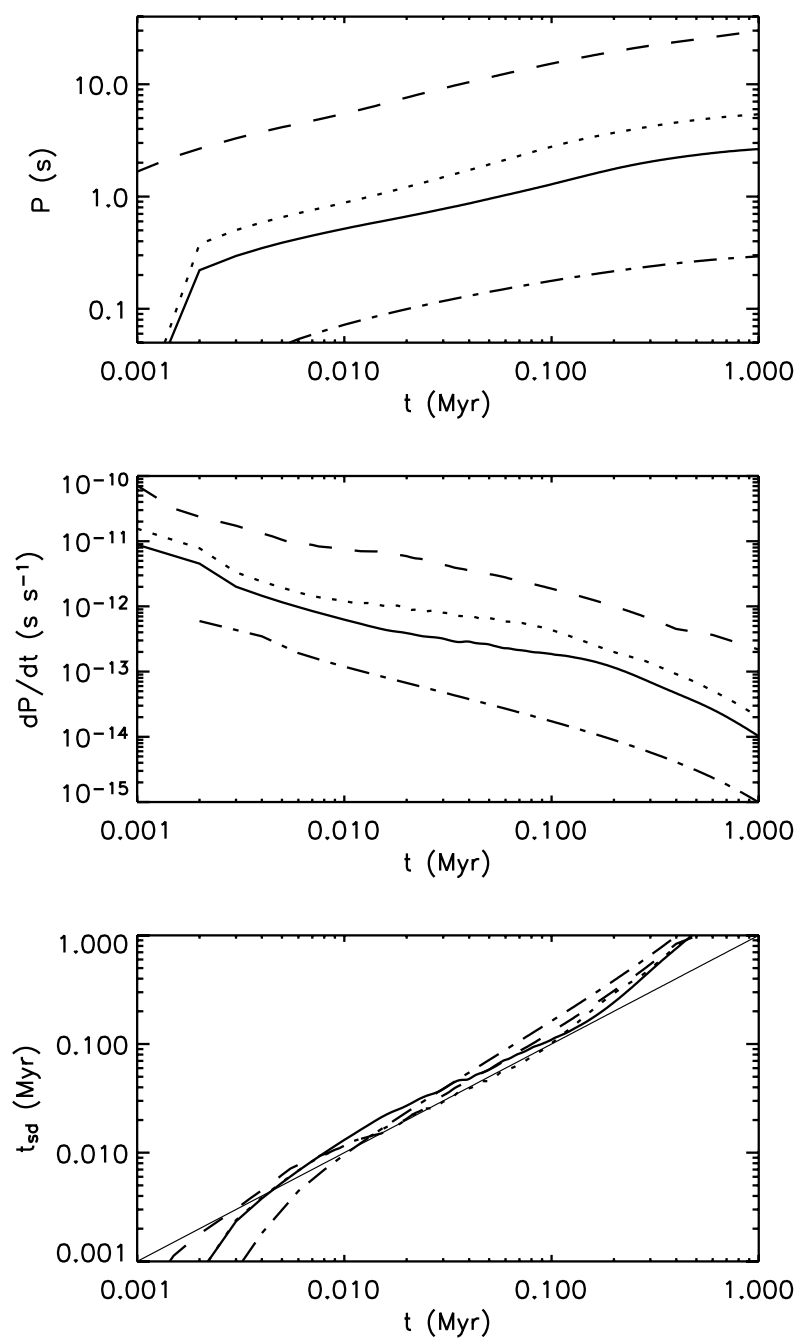

Fig. 9. Evolution of the period (top), period derivative (middle), and spin-down age (bottom) in four selected models with different initial dipolar field strengths. The line-styles correspond to models A (solid lines), D (dash-dotted line), E (dots), and G (dashes).

distribution of middle-aged NSs, which could be more complex than previously thought. Such complex field structures and the

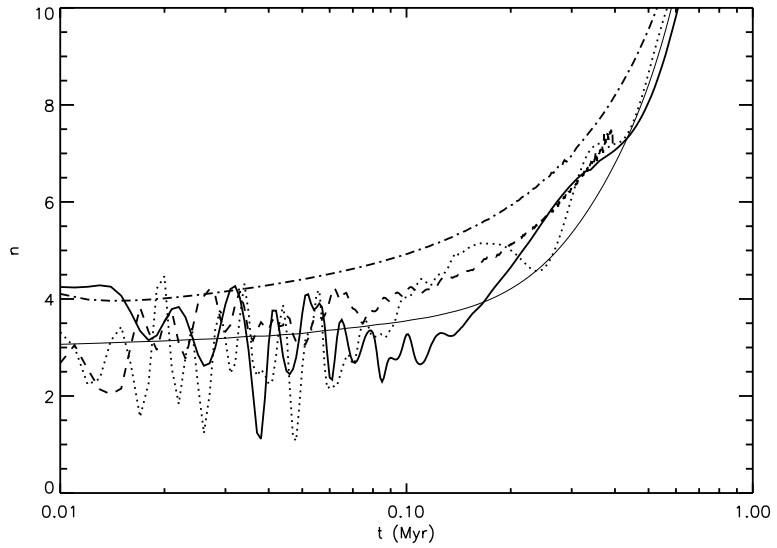

Fig. 10. Evolution of the braking index $n=v \ddot{v} / \dot{v}^{2}$ for the same models as in Fig. 9.

local deposition of energy by Joule heating favor surface temperature distributions characterized not only by two hot polar caps, but for example by a hot equatorial belt, as has probably been seen in RX J0720.4-3125 (Haberl et al. 2006; Pérez-Azorín et al. 2006b) or can be inferred from the light curve of RBS 1223 (Schwope et al. 2005).

Though the toroidal part of the crustal field undergoes a spectacular dissipation and rearrangement, the coupling between both parts also affects the evolution of the poloidal field, which is responsible for the spin down of NSs. Thus, the complex interaction of Hall drift and Ohmic dissipation is also reflected in the temporal behavior of the braking index and, in principle, accessible for observations. Our models show that the braking index of young ( $\$ 10^{5}$ years), magnetized NSs exhibits a very wild variation and can reach any value from 1.5 to 4 . After the Hall stage, when Ohmic dissipation controls the evolution and the dipolar magnetic field decreases steadily, the braking index gradually increases and might reach very high values. This process will cease after the crustal field is dissipated almost completely, or when the conductivity increases as the NS cools down. During the photon cooling era, the braking index will approach its canonical (dipolar) value of 3 again.

If the initial magnetic field is too weak $\left(\lesssim 10^{12} \mathrm{G}\right)$ for the Hall stage to be relevant, the evolution will proceed according to purely Ohmic field decay. The very existence of magnetars and of their presumable descendants, thermally emitting NSs with 
$B \approx 10^{13} \mathrm{G}$, suggests that the fraction of NSs born with large magnetic fields may be greater than expected.

Acknowledgements. We thank J.A. Miralles, M. Rheinhardt, A. Reisennegger, J.M. Ibáñez and D.N. Aguilera for useful comments and critical discussions. This work has been supported by the Spanish Ministerio de Ciencia y Tecnología grant AYA 2004-08067-C03-02. JAP is supported by a Ramón y Cajal contract from the Spanish MEC. UG acknowledges support from the Spanish MEC (grant SAB-2005-0122).

\section{Appendix A: Induction equation in cylindrical coordinates}

In this appendix we use a different set of variables to derive the induction equation in cylindrical coordinates $(R \equiv r \sin \theta, \varphi, z \equiv$ $r \cos \theta$ ) and use the notation of Hollerbach \& Rüdiger (2002). Alternatively to our decomposition in Eq. (3), one can in axial symmetry simply work with the $\varphi$-components of the vector potential and of the magnetic field,

$\boldsymbol{B}=\boldsymbol{B}_{\mathrm{pol}}+\boldsymbol{B}_{\mathrm{tor}}=\nabla \times\left(A_{\varphi} \boldsymbol{e}_{\varphi}\right)+B_{\varphi} \boldsymbol{e}_{\varphi}$.

In cylindrical coordinates, we can write explicitly the different components of the magnetic field and the current density:

$\boldsymbol{B}=-\frac{\partial A_{\varphi}}{\partial z} \boldsymbol{e}_{R}+\frac{1}{R} \frac{\partial\left(R A_{\varphi}\right)}{\partial R} \boldsymbol{e}_{z}+B_{\varphi} \boldsymbol{e}_{\varphi}$

$\frac{4 \pi}{c} \boldsymbol{J}=\nabla \times \boldsymbol{B}=-\frac{\partial B_{\varphi}}{\partial z} \boldsymbol{e}_{R}+\frac{1}{R} \frac{\partial\left(R B_{\varphi}\right)}{\partial R} \boldsymbol{e}_{z}+\hat{J}_{\varphi} \boldsymbol{e}_{\varphi}$,

where we have introduced the notation

$\hat{J}_{\varphi} \equiv-\nabla^{2} A_{\varphi}+\frac{A_{\varphi}}{R^{2}}$.

The induction equation in terms of this variables reads:

$$
\begin{aligned}
\frac{\partial A_{\varphi}}{\partial t}= & -\frac{\hat{\tau}}{\hat{\sigma}} \boldsymbol{e}_{\varphi} \cdot\left[\left(\nabla \times \boldsymbol{B}_{\mathrm{tor}}\right) \times \boldsymbol{B}_{\mathrm{pol}}\right]-\frac{\hat{J}_{\varphi}}{\hat{\sigma}} \\
\frac{\partial B_{\varphi}}{\partial t}= & \boldsymbol{e}_{\varphi} \cdot \nabla \times\left(\frac{\hat{\tau}}{\hat{\sigma}}\left[\left(\nabla \times \boldsymbol{B}_{\mathrm{pol}}\right) \times \boldsymbol{B}_{\mathrm{pol}}+\left(\nabla \times \boldsymbol{B}_{\mathrm{tor}}\right) \times \boldsymbol{B}_{\mathrm{tor}}\right]\right) \\
& +\nabla \times\left[\frac{1}{\hat{\sigma}} \nabla \times B_{\varphi}\right] .
\end{aligned}
$$

The term appearing in the r.h.s. of the first equation can be written as follows:

$\boldsymbol{e}_{\varphi} \cdot\left[\left(\nabla \times \boldsymbol{B}_{\text {tor }}\right) \times \boldsymbol{B}_{\mathrm{pol}}\right]=\frac{1}{R^{2}}\left[\nabla\left(R A_{\varphi}\right) \times \nabla\left(R B_{\varphi}\right)\right]_{\varphi}$.

The two terms inside the curl of the second equation are

$$
\begin{aligned}
& \left(\nabla \times \boldsymbol{B}_{\text {tor }}\right) \times \boldsymbol{B}_{\text {tor }}=-\frac{B_{\varphi}}{R} \nabla\left(R B_{\varphi}\right) \\
& \left(\nabla \times \boldsymbol{B}_{\text {pol }}\right) \times \boldsymbol{B}_{\text {pol }}=\frac{J_{\varphi}}{R} \nabla\left(R A_{\varphi}\right) .
\end{aligned}
$$

Then, taking the curl and after some algebra, the two terms appearing in the equation for $B_{\varphi}$ can be written as follows:

$$
\begin{aligned}
\boldsymbol{e}_{\varphi} \cdot \nabla \times\left[\left(\nabla \times \boldsymbol{B}_{\text {tor }}\right) \times \boldsymbol{B}_{\text {tor }}\right] & =\left[\nabla\left(R B_{\varphi}\right) \times \nabla\left(B_{\varphi} / R\right)\right]_{\phi} \\
& =-\frac{2 B_{\varphi}}{R} \frac{\partial B_{\varphi}}{\partial z} \\
\boldsymbol{e}_{\varphi} \cdot \nabla \times\left[\left(\nabla \times \boldsymbol{B}_{\mathrm{pol}}\right) \times \boldsymbol{B}_{\mathrm{pol}}\right] & =-\left[\nabla\left(R A_{\varphi}\right) \times \nabla\left(\hat{J}_{\varphi} / R\right)\right]_{\phi},
\end{aligned}
$$

where the first one is used in the derivation of Eq. (27).

\section{References}

Baym, G., Pethick, C. J., \& Pines, D. 1969, Nature, 224, 674

Baym, G., Pethick, C. J., \& Sutherland, P. 1971, ApJ, 170, 299

Braithwaite, J. 2006, A\&A, 453, 687

Cumming, A., Arras, P., \& Zweibel, E. 2004, ApJ, 609, 999

Douchin, F., \& Haensel, P. 2001, A\&A, 380, 151

Geppert, U., \& Wiebicke, H.-J. 1991, A\&AS, 87, 217

Geppert, U., \& Rheinhardt, M. 2002, A\&A, 392, 1015

Geppert, U., Küker, M., \& Page, D. 2004, A\&A, 426, 267

Geppert, U., Küker, M., \& Page, D. 2006, A\&A, 457, 937

Gil, J., Melikidze, G., \& Geppert, U. 2003, A\&A, 407, 315

Gil, J., Melikidze, G., \& Zhang, B. 2006, ApJ, 650, 1048

Goldreich, P., \& Reisenegger, A. 1992, ApJ, 95, 250

Gonthier, P., van Guilder, R., \& Harding, A. 2004, ApJ, 604, 775

Haberl, F., Turolla, R., de Vries, C., et al. 2006, A\&A, 451, L17

Haensel, P., Urpin, V., \& Yakovlev, D. 1990, A\&A, 229, 133

Harding, A. K., \& Lai, D. 2006, Rep. Prog. Phys., 69, 2631

Hartman, J., Bhattacharya, D., Wijers, R., \& Verbunt, F. 1997, A\&A, 322, 477

Hollerbach, R., \& Rüdiger, G. 2002, MNRAS, 337, 216

Johnston, S., \& Galloway, D. 1999, MNRAS, 306, L50

Jones, P. B. 2004, Phys. Rev. Lett., 93, 221101

Konenkov, D., \& Geppert, U. 2001, MNRAS, 325, 426

Muslimov, A. 1994, MNRAS, 267, 523

Naito, T., \& Kojima, Y. 1994, MNRAS, 266, 597

Page, D., Geppert, U., \& Weber, F. 2006, Nucl. Phys. A, 777, 497

Pérez-Azorín, J. F., Miralles, J. A., \& Pons, J. A. 2006a, A\&A, 451, 1009

Pérez-Azorín, J. F., Pons, J. A., Miralles, J. A., \& Miniutti, G. 2006b, A\&A, 459, 175

Pons, J. A., Link, B., Miralles, J. A., \& Geppert, U. 2007, Phys. Rev. Lett., 98, 071101

Pons, J. A., Walter, F. M., Lattimer, J. M., et al. 2002, ApJ, 564, 981

Potekhin, A. Y. 1999, A\&A, 351, 787

Rädler, K. H. 1973, Astron. Nachr., 294, 213

Regimbau, T., \& de Freitas Pacheco, J. 2001, A\&A, 374, 182

Reisenegger, A., Prieto, J. P., Benguria, R., Lai, D., \& Araya, P. A. 2005, in Magnetic Fields in the Universe: From Laboratory and Stars to Primordial Structures, AIP Conf. Proc., 784, 263

Rheinhardt, M., \& Geppert, U. 2002, Phys. Rev. Lett., 88, 101103

Schwope, A., Hambaryan, V., Haberl, F., \& Motch, C. 2005, A\&A, 441, 597

Shalybkov, D., \& Urpin, V. 1997, A\&A, 321, 685

Urpin, V., \& Shalybkov, D. 1995, A\&A, 294, 117

Vainshtein, S. I., Chitre, S. I., \& Olinto, A. V. 2000, Phys. Rev. E, 61, 4422

van Adelsberg, M., Lai, D., \& Potekhin, A. 2005, ApJ, 628, 902

Wiebicke, H.-J., \& Geppert, U. 1995, A\&A, 294, 303

Woods, P., \& Thompson, C. 2006, in Compact Stellar X-ray Sources, ed. W. H. G. Lewin, \& M. van der Klis (Cambridge University Press), 547

Yakovlev, D., \& Shalybkov, D. 1991, Ap\&SS, 176, 191 